\title{
artigo
}

\section{Fisioterapeuta e NASF: conhecimentos de graduados da primeira e segunda década do século XXI}

\author{
Physiotherapy and NASF: knowledge of graduates from the first and second decade of the XXI century
}

Fisioterapia y NASF: conocimiento de los graduados de la primera y segunda década del siglo XXI

\begin{abstract}
RESUMO
Objetivo: Verificar o conhecimento entre profissionais formados na $1^{\text {a }}$ e na $2^{\text {a }}$ década do século XXI quanto as atribuições do fisioterapeuta no núcleo de apoio à saúde da família. Método: Estudo quantitativo, transversal, composto por 35 fisioterapeutas do município de Porto Velho, com registro ativo, divididos em dois grupos ( $1^{\mathrm{a}}$ e $2^{\mathrm{a}}$ década). Os profissionais responderam um questionário com perguntas dicotômicas acerca da atuação do fisioterapeuta através da plataforma Google Forms. Resultados: Não se verificou significância estatística entre os grupos, contudo, em uma discussão mais sintética de forma descritiva de percentuais decorrente das diferenças de amostras nos grupos, facilitando o risco de viés, verificou-se que o grupo da $2^{\mathrm{a}}$ década detém mais conhecimento em relação ao outro grupo. Conclusão: Durante a formação acadêmica existem disciplinas voltadas para a atuação na atenção básica, porém o foco na área terciária, possibilita que grande parte dos profissionais atrelem suas atuações ao papel reabilitador.
\end{abstract}

DESCRITORES: Fisioterapia; Atenção Primária à Saúde; Estratégia Saúde da Família; Conhecimento.

\section{ABSTRACT}

Objective: To verify the knowledge among professionals trained in the 1st and 2nd decades of the XXI century regarding the physiotherapist's duties in the family health support center. Method: Quantitative, cross-sectional study, comprising 35 physiotherapists from the city of Porto Velho, with active registration, divided into two groups (1st and 2nd decade). The professionals answered a questionnaire with dichotomous questions about the physiotherapist's performance through the Google Forms platform. Results: There was no statistical significance between the groups, however, in a more synthetic discussion in a descriptive form of percentages due to the differences in samples in the groups, facilitating the risk of bias, it was found that the 2nd decade group has more knowledge in relation to the other group. Conclusion: During academic training there are disciplines aimed at working in primary care, but the focus on the tertiary area, allows a large part of professionals to link their work to the rehabilitation role.

DESCRIPTORS: Physiotherapy; Primary Health Care; Family Health Strategy; Knowledge;

\section{RESUMEN}

Objetivo: Verificar el conocimiento de los profesionales formados en la $1^{\text {a }}$ y $2^{\text {a }}$ décadas del siglo XXI sobre las funciones del fisioterapeuta en el centro de apoyo a la salud familiar. Método: Estudio cuantitativo, transversal, compuesto por 35 fisioterapeutas de la ciudad de Porto Velho, con registro activo, divididos en dos grupos ( $1^{\mathrm{a}}$ y $2^{\mathrm{a}}$ década). Los profesionales respondieron un cuestionario con preguntas dicotómicas sobre el desempeño del fisioterapeuta a través de la plataforma Google Forms. Resultados: No hubo significación estadística entre los grupos, sin embargo, en una discusión más sintética en forma descriptiva de porcentajes debido a las diferencias en las muestras en los grupos, facilitando el riesgo de sesgo, se encontró que el grupo de 2da década tiene más conocimiento en relación con el otro grupo. Conclusión: Durante la formación académica existen disciplinas orientadas a trabajar en atención primaria, pero el enfoque en el área terciaria, permite a una gran parte de los profesionales vincular su trabajo con el rol rehabilitador.

DESCRIPTORES: Fisioterapia; Atención Primaria de Salud; Estrategia de Salud de la Familia; Conocimiento.

RECEBIDO EM: 15/12/2020 APROVADO EM: 08/01/2021

\author{
Fábio Bruno de Souza Santos
}

Graduado em Fisioterapia (Faculdade Interamericana de Porto Velho/UNIRON) 2020.

ORCID: 0000-0003-4521-6879 


\section{Samuel da Silva Souza}

Graduado em Fisioterapia (Faculdade Interamericana de Porto Velho/UNIRON) 2020.

ORCID: 0000-0002-0325-6525

\section{Geiferson Santos do Nascimento}

Graduado em Fisioterapia (Faculdade Interamericana de Porto Velho/UNIRON) 2016; Especialista em Ortopedia e Traumatologia (Faculdade Interamericana de Porto Velho/UNIRON) 2017; Especialista em Fisioterapia Neurofuncional Adulto (UNIMINAS) 2020; Especialista em Serviço Social e Saúde Coletiva (ESTRATEGO) 2020; Mestrando em Psicologia (UNIR) 2019.

ORCID: 0000-0003-1726-0936

\section{Isabella Naiara de Almeida}

Graduada em Fisioterapia (Faculdade São Lucas) 2007; Especialista em Fisioterapia Hospitalar (UNOESTE) 2009; Especialista em Gestão Pedagógica do Ensino Superior (Faculdade Interamericana de Porto Velho/UNIRON) 2013; Mestre em Terapia Intensiva (SOBRATI) 2017.

ORCID: 0000-0003-0853-9150

\section{INTRODUÇÃO}

$\mathbf{N}$ o Brasil, a Atenção Primária à Saúde (APS) passou a ser notada em 1994 com a criação do Programa de Saúde da Família (PSF) para reorganizar e reorientar as ações da atenção básica, assim em 2006 passou a ser conhecida como Estratégia Saúde da Família (ESF). Diante da extensão dos atendimentos e das dificuldades na interdisciplinaridade durante as ações da ESF, o Núcleo de Apoio à Saúde da Família (NASF) é criado pela Portaria GM n ${ }^{\circ} 154$, de 24 de janeiro de 2008, para contribuir com a atenção e a gestão da saúde na atenção básica. $\mathrm{O}$ NASF, foi criado com objetivo de tornar maior a atuação da $\mathrm{AB}$ sob novas perspectivas de atuação do ESF, dando maior resolutividade e qualidades da atenção à saúde. Dentre os profissionais que compõem o NASF, o fisioterapeuta é um deles, haja vista ser um profissional capacitado para atuar no campo da promoção em saúde, prevenção, tratamento e reabilitação ${ }^{(1)}$.

Fonseca ${ }^{(2)} \mathrm{em}$ uma revisão integrativa da literatura constatou que há uma diversidade em relação ao público-alvo e a atuação do fisioterapeuta no NASF, em que há um redirecionamento da sua atuação para atenção terciária devido as condições físicas e econômicas inadequadas, e a falta de conhecimento dos membros da equipe e dos usuários.

Desde o reconhecimento da profissão o fisioterapeuta concentrou sua assistência exclusivamente no tratamento de doenças,
No Brasil, a Atenção

Primária à Saúde

(APS) passou a ser

notada em 1994

com a criação do

Programa de Saúde

da Família (PSF)

para reorganizar e

reorientar as ações da

atenção básica, assim

em 2006 passou a

ser conhecida como

Estratégia Saúde da

Família (ESF). impulsionado pelo contexto histórico da criação da profissão através do decreto-lei $938 / 69$, ao definir como atividade privativa do fisioterapeuta, executar métodos e técnicas com a finalidade de restaurar, desenvolver e conservar a capacidade física do paciente ${ }^{(3)}$. Com a necessidade de rever o perfil profissional dentro da Atenção Básica (AB) surge no ano de 2002 a diretriz curricular básica do curso de fisioterapia, publicada pelo Conselho Nacional de Educação, definindo como habilidades e competências ao final da graduação, como por exemplo, a atuação em todos os níveis de atenção à saúde ${ }^{(4)}$. Contudo, mesmo com as diretrizes curriculares de 2002, foi somente após a criação do NASF em 2008, que o caminho da formação acadêmica começou a ter direcionamentos para a atuação junto à $A B$. Entretanto, poucos profissionais atuam ou conhecem suas atribuições na $\mathrm{AB}$, que vão além da reabilitação físico-funcional, uma vez que a formação profissional esteve voltada por muito tempo para a execução de técnicas com a finalidade de restaurar a capacidade física do paciente e a portaria $\mathrm{n}^{\circ} 154$ de 2008 , cuja sua concepção delimita aos gestores públicos municipais da administração pública a oportunidade e conveniência em implementar a utilização da prática fisioterapêutica no NASF ${ }^{(5 ;(6)}$.

Dessa forma, objetivou-se verificar se há discrepância no conhecimento entre os profissionais formados na primeira e segunda década do século XXI e a pergunta norteadora da pesquisa foi: Existe dife- 
rença de conhecimentos acerca de atuação fisioterapêutica na atenção básica entre os profissionais formados antes e pós Portaria GM nº 154/2008 - Ministério da Saúde?

\section{MÉTODO}

Trata-se de um estudo de abordagem quantitativa, do tipo descritivo e caráter transversal ${ }^{(7)}$.

O universo do estudo foi composto por 35 (trinta e cinco) fisioterapeutas, divididos em dois grupos, selecionados conforme o período de formação, sendo um grupo composto por 11 (onze) profissionais, sendo 2 (dois) do sexo masculino e 9 (nove) do sexo feminino, com formação concluída entre o mês de janeiro do ano de 2000 ao mês de dezembro do ano de 2008 e outro grupo formado por 24 (vinte e quatro) profissionais, sendo 6 (seis) do sexo masculino e 18 (dezoito) do sexo feminino, com formação concluída entre o mês de janeiro do ano de 2009 ao mês de dezembro do ano de 2018.

Ressalta-se que os profissionais inclu-

\section{Tabela 1. Frequência absoluta das respostas referentes às 15 questões de} ambos os grupos.

\section{QUESTÕES/ GRUPOS}

Questão 1
Questão 2
Questão 3
Questão 4
Questão 5
Questão 6
Questão 7
Questão 8
Questão 9
Questão 10
Questão 11
Questão 12
Questão 13
Questão 14
Questão 15
P=signifiância.
Fonte: Próprio autor

ídos na pesquisa tiveram como critérios serem registrados no CREFITO ${ }^{(18)}$, terem atuado no ramo de fisioterapia com

\section{Ressalta-se que os profissionais incluídos na}

pesquisa tiveram

como critérios serem registrados no CREFITO ... período mínimo de 06 (seis) meses, e que estiveram presentes no centro urbano do município de Porto Velho (RO) no período da coleta de dados.

Foram subtraídos aqueles que não atenderam aos requisitos necessários para a formalização da pesquisa acadêmica e aqueles que não se encontravam nos locais visitados para coleta de dados, além daqueles que se encontravam incapacitados fisicamente e/ou mentalmente devido a lembranças passadas, cansaço ou até mesmo devido experiências negativas acerca do tema.

Os pesquisadores foram ao encontro dos profissionais a partir da pesquisa por hospitais públicos e da iniciativa privada, clínicas de fisioterapias, unidades básicas de saúde no período de outubro e novembro de 2019. Utilizou-se como ferramenta de coleta um celular Samsung J5 Prime, conectando-se aos servidores Google que disponibilizam de forma gratuita o aplicativo Google Forms, destinado a coleta de dados e geração de gráficos/tabelas. Os participantes aceitaram participar da pesquisa através do Termo de Consentimento Livre e Esclarecido (TCLE) e acessaram o questionário de atuação no NASF (ANEXO I). A pesquisa seguiu os requisitos da Resolução no 466/2012CNS e foi aprovada pelo Comitê de Ética e Pesquisa da Faculdade Integradas Aparício Carvalho (FIMCA) sob o $\mathrm{n}^{\circ}$ 54669416.1.0000.0012.

Os dados foram testados quanto a distribuição de normalidade através do teste Shapiro Wilk e para análise dos dados as variáveis categóricas foram apresentadas em frequências absolutas e relativas, utilizado para comparação entre os grupos da primeira e segunda década o Teste do Qui-quadrado de Pearson. As análises foram realizadas no programa SPPS 22.0, e a significância estatística foi estipulada em 5\%.

\section{RESULTADOS}

A análise das variáveis categóricas após a estatística, demonstrou a não existência de significância entre os grupos, conforme apresentado na tabela 1. 
Para uma efetiva discussão dos temas abordados dentro dos questionários, as perguntas foram divididas por subgrupos e após isso agrupadas para realização de uma análise mais sintética dos resultados obtidos em forma de percentuais (\%).

\section{DISCUSSÃO}

Analisando a Tabela 2, os percentuais dos grupos, percebe-se que os profissionais com formação posterior ao ano de 2008, apresentam maior conhecimento sobre a inserção do fisioterapeuta no NASF, bem como ainda a importância deste profissional neste nível de atenção.

Tratando-se de uma profissão em construção, completando no ano de 2019, 50 anos, mostra que os fisioterapeutas passam por constantes transformações. Umas destas transformações é a inserção do fisioterapeuta dentro do APS desde o ano de 2008, através da regulamentação do NASF ${ }^{(8)}$.

O próprio conselho de classe no ano de 2009, através da Resolução no 363/2009 reconhece a Saúde coletiva como espe-

Tabela 2. Frequência relativa em relação as questões que abordam a inserção do fisioterapeuta no NASF.

\begin{tabular}{lcccc}
\multicolumn{1}{c}{ QUESTÕES/GRUPOS } & GRUPO $2000-2008(1)$ & \multicolumn{2}{c}{ GRUPO $2009-2018$ (2) } \\
& SIM & NÃO & SIM & NÃO \\
Questão 2 & $100 \%$ & $0 \%$ & $100 \%$ & $0 \%$ \\
Questão 4 & $57,1 \%$ & $42,9 \%$ & $50 \%$ & $50 \%$ \\
Questão 6 & $88,7 \%$ & $14,3 \%$ & $83,3 \%$ & $16,7 \%$ \\
Fonte: Próprio Autor & & & & \\
\hline
\end{tabular}

Tabela 3. Frequência relativa em relação as questões que abordam a atuação do fisioterapeuta no NASF.

\begin{tabular}{lcccc}
\multicolumn{1}{c}{ QUESTÕES/GRUPOS } & GRUPO & 2000-2008 (1) & \multicolumn{2}{c}{ GRUPO 2009-2018 (2) } \\
& SIM & NÃO & SIM & NÃO \\
Questão 3 & $28,6 \%$ & $71,4 \%$ & $94,4 \%$ & $5,6 \%$ \\
Questão 5 & $100 \%$ & $0 \%$ & $94,4 \%$ & $5,6 \%$ \\
Questão 8 & $100 \%$ & $0 \%$ & $77,8 \%$ & $22,2 \%$ \\
Questão 9 & $85,7 \%$ & $14,3 \%$ & $83,3 \%$ & $16,7 \%$ \\
Questão 10 & $28,6 \%$ & $71,4 \%$ & $77,8 \%$ & $22,2 \%$ \\
Questão 11 & $100 \%$ & $0 \%$ & $94,4 \%$ & $5,6 \%$ \\
Questão 12 & $71,4 \%$ & $28,6 \%$ & $55,6 \%$ & $44,4 \%$ \\
Fonte: Próprio Autor & & & &
\end{tabular}

Tabela 4. Frequência relativa em relação as questões que abordam a formação acadêmica para atuação no NASF.

\begin{tabular}{lcccc}
\multicolumn{1}{c}{ QUESTÕES/GRUPOS } & \multicolumn{2}{c}{ GRUPO $2000-2008$ (1) } & \multicolumn{2}{c}{ GRUPO $2009-2018$ (2) } \\
& SIM & NÃO & SIM & NÃO \\
Questão 1 & $20 \%$ & $17,1 \%$ & $11,4 \%$ & $51,4 \%$ \\
Questão 7 & $100 \%$ & $0 \%$ & $88,9 \%$ & $11,1 \%$ \\
Questão 13 & $57,1 \%$ & $42,9 \%$ & $83,3 \%$ & $16,7 \%$ \\
Questão 14 & $28,6 \%$ & $71,4 \%$ & $16,7 \%$ & $83,3 \%$ \\
Questão 15 & $85,7 \%$ & $14,3 \%$ & $88,9 \%$ & $11,1 \%$ \\
Fonte: Próprio Autor & & & &
\end{tabular}

cialidade da fisioterapia, desmistificando a ideia de um profissional totalmente formado para o papel reabilitador ${ }^{(9)}$.

Contudo, há resistência da atuação do profissional na $\mathrm{AB}$ advinda da formação acadêmica, dificultando o acesso ao serviço e o conhecimento tanto dos próprios fisioterapeutas como da sociedade acerca das atribuições do profissional.

Ao analisar os resultados do segundo agrupamento de questões, percebe-se uma diferença entre as opiniões dos grupos, onde o grupo 2, grande parte dos participantes demonstraram conhecimentos sobre a atuação e atribuições do fisioterapeuta dentro APS, destoando do encontrado no grupo 1, contudo grande parte ainda verifica essas atribuições e funções vinculadas ao papel de um profissional reabilitador.

$\mathrm{O}$ conhecimento das atribuições e competências do profissional na APS também vem sendo difundido nas instituições de níveis superiores, visto que mesmo com a previsão nas Diretrizes Curriculares do Curso ${ }^{(10)}$, somente após a criação do NASF as instituições de ensino passaram a fomentar esse campo de atuação, facilitando assim que os profissionais formados após esse período sejam detentores de maiores conhecimentos sobre sua atuação neste nível de saúde ${ }^{(11)}$.

Verificando os resultados, percebeu-se em ambos os grupos que existem uma concordância que durante o processo de formação existe disciplinas voltadas para atuação do fisioterapeuta na APS, porém com foco reabilitador. Apresentaram ainda similaridade de pensamentos quanto ao seu preparo para atuação neste nível em saúde e que a modalidade de ensino em EAD na fisioterapia não contribui na formação deste profissional para o nível de área primária.

Os avanços em ensino de fisioterapia na APS tiveram grandes resultados, principalmente com a abertura de campos de estágios para as vivências práticas desta realidade de atuação ${ }^{(12)}$. Essas vivências podem ser camufladas ou até extintas com o surgimento do EAD onde a ausência de contato direto com paciente, disciplinas com maior enfoque teórico do que prático, pode abrir 


\section{artigo}

Santos, F.B.S.; Souza, S.S.; Nascimento, G.S.; Almeida, I.N.

Fisioterapeuta e NASF: conhecimentos de graduados da primeira e segunda década do século XXI

novos precedentes para que essa área de atuação ainda em crescimento, possa ter todo seu potencial minimizado ou até mesmo defasado decorrente de uma formação incompleta de novos profissionais ${ }^{(13)}$.

\section{CONCLUSÃO}

O resultado estatístico do estudo demonstrou a não existência de significância entre os grupos, contudo, em uma análise mais sintética dos resultados em forma de frequências absolutas, percebeu-se diferenças de saberes entre os participantes da pesquisa em relação a inserção, a importância do fisioterapeuta e suas atribuições no NASF, sendo que muitas vezes essas atribuições não definidas em portaria ministerial, são executadas de forma de apoio matricial, desenvolvidas em condição de gestão das ESF e possibilitando uma abordagem multidisciplinar.

Mesmo diante das divergências encontradas entre os grupos, ainda assim, foi possível verificar uma similaridade de conhecimento em relação ao perfil de formação, em que mesmo que haja a existência de disciplinas voltadas para atuação na APS, os participantes ainda vinculam as atribuiçõos e funções deste profissional a reabilitação individual, demonstrando de forma clara que a visão do profissional neste campo de atuação continua de forma engessada, com avanços sobre conhe- cimentos básicos da possibilidade de existência profissional, porém com limitações sobre as atuações e habilidades destinadas ao fisioterapeuta.

Contudo, verifica-se que essa atuação do fisioterapeuta na APS vem passando por uma evolução constante, uma vez que há uma desconstrução do perfil reabilitador após a inclusão deste profissional no NASF, para isso é necessário avanços científicos que demonstrem de forma estatística as abordagens e eficácia deste profissional na APS, estudos que visam ampliar as melhorias na qualidade da formação acadêmica, tendo como consequência a promoção de melhores experiências e vencer paradigmas ainda existentes. -

\section{REFERÊNCIAS}

1. Fernandes SC, Ros MA. Desconstruir para transformar: o perfil do fisioterapeuta para o Núcleo de Apoio à Saúde da Família. Fisioterapia Brasil, 2018;19(2):249-25. Disponível em: http://docs. bvsalud.org/biblioref/2018/08/911298/desconstruir-para-transformar-o-perfil-do-fisioterapeuta-para-o_gsCUXAj.pdf

2. Fonseca JMA, Rodrigues MTP, Mascarenhas MDM, LIMA LHO. A fisioterapia na atenção primária à saúde: uma revisão integrativa. Revista Brasileira em Promoção da Saúde, Fortaleza, 29(2): 288294, abr./jun., 2016. Disponível em https://periodicos.unifor.br/ RBPS/article/view/4593/pdf

3. Bispo JPJ. Fisioterapia e saúde coletiva: desafios e novas responsabilidades profissionais. Ciênc. saúde coletiva, Rio de Janeiro, v. 15, supl. 1, p. 1627-1636, Junho 2010.Disponivel em: http://www.scielo.br/scielo.php?script=sci_arttext\&pid=S1413-81232010000700074\&Ing=en\&nrm=iso

4. Brasil. Ministério da Educação. Conselho Nacional de Educação. Câmara de Educação Superior. Resolução CNE/CES n 4, de 19 de fevereiro de 2002. Institui Diretrizes Curriculares Nacionais do Curso de Graduação em Fisioterapia. Diário Oficial da União, Brasilia, 4 de março de 2002. Seção 1, p. 11. Disponivel em: http://portal.mec. gov.br/cne/arquivos/pdf/CES042002.pdf

5. Braghini CC, Ferretti F, Ferraz L. Atuação do fisioterapeuta no contexto dos núcleos de apoio à saúde da família. Fisioterapia em movimento. Curitiba, v. 30, n. 4, p. 703-713, Dec. 2017 Disponivel em: http://www.scielo.br/scielo.php?script=sci_arttext\&pid=S0103-51502017000400703

6. Conselho Federal de Fisioterapia e Terapia ocupacional (COFFITO). Resolução COFFITO n³ 363, de 20 de maio de 2009. Reconhece a Fisioterapia em Saúde Coletiva como especialidade do profissional Fisioterapeuta e dá outras providências. Brasilia: COFFITO, 2014. Disponível em: https://www.coffito.gov.br/nsite/?p=3126

7. Fontelles JM, Simoes MG, Farias SH, Fontenelle RGS. Metodologia da pesquisa científica: diretrizes para a elaboração de um protocolo de pesquisa. Núcleo de Bioestatística Aplicado à pesquisa da Universidade da Amazônia, UNAMA, 2009. Disponível em: https://cienciassaude.medicina.ufg.br/up/150/o/Anexo_C8_NONAME.pdf

8. Brasil. Ministério da Saúde. Portaria Nº 154, de 24 de janeiro de 2008. Cria os Núcleos de Apoio à Saúde da Família (NASF). Disponivel em: http://bvsms.saude.gov.br/bvs/saudelegis/gm/2008/ prt0154_24_01_2008.html

9. Conselho Federal de Fisioterapia e Terapia ocupacional (COFFITO). Resolução COFFITO n 363, de 20 de maio de 2009. Reconhece a Fisioterapia em Saúde Coletiva como especialidade do profissional Fisioterapeuta e dá outras providências. Brasília: COFFITO, 2014. Disponivel em: https://www.coffito.gov.br/nsite/?p=3126

10. Formiga NFB, Ribeiro KSQS. A inserção do fisioterapeuta na atenção básica: uma analogia entre as experiências acadêmicas e a proposta dos Núcleos de Apoio à Saúde da Familia (NASF). Revista Brasileira de Ciências da Saúde. Disponivel em: https://periodicos. ufpb.br/ojs2/index.php/rbcs/article/view/10639

11. Bispo JPJ. Formação em fisioterapia no Brasil: reflexões sobre a expansão do ensino e os modelos de formação. História, Ciências, Saúde. Rio de Janeiro, 2009. Disponível em: http://www.scielo.br/ scielo.php?script=sci_arttext\&pid=S0104-59702009000300005

12. Seriano KN, Muniz VRC, Carvalho, MEIM. Percepção de estudantes do curso de fisioterapia sobre sua formação profissional para atuação na atenção básica no Sistema Único de Saúde. Fisioterapia e pesquisa. 2013. Disponível em: http://www.scielo.br/ scielo.php?pid=S1809-29502013000300009\&script=sci_abstract\&tlng=pt

13. Brenner F, Pianesser R, Espindola, DB; Pinto I, Musa M, Gouvêa A, et all. Revisão Sistemática da Educação a Distância: um estudo de caso da EAD no Brasil. ESUD, 2014 - XI Congresso Brasileiro de Ensino Superior à Distância. Florianópolis, 05-08 de agosto de 2014. UNIREDE. Disponivel em: http://esud2014.nute.ufsc.br/ anais-esud2014/files/pdf/128187.pdf 


\section{ANEXO I}

\section{Questionário}

1. Você conhece o NASF (Núcleo de Apoio à Saúde da Família)?
( ) Sim
( ) Não

Se a resposta for afirmativa, continue.

2. O fisioterapeuta é um profissional importante na composição do NASF, uma vez que o núcleo atua na atenção básica à saúde?
( ) Sim
( ) Não

3. O fisioterapeuta dentro das normativas do NASF atua somente na reabilitação física e no preparo para o parto?
( ) Sim
( ) Não

4. Antes da criação do NASF, o fisioterapeuta podia atuar na atenção básica?
( ) Sim
( ) Não

5. O fisioterapeuta dentro do NASF participa da discussão de casos clínicos com os demais profissionais do núcleo?
( ) $\operatorname{Sim}$
( ) Não

6. Com a criação do NASF no ano de 2008, o profissional conseguiu maior amparo e visibilidade para atuar na atenção básica?
( ) Sim

( ) Não

7. Durante sua formação acadêmica, teve disciplinas que abordaram a atuação do fisioterapeuta na atenção básica?
( ) Sim
( ) Não

8. Dentro do NASF, o fisioterapeuta participa do planejamento coletivo das ações do núcleo?
( ) Sim
( ) Não

9. Palestras e orientações para educação permanente da população são de competências dos fisioterapeutas?
( ) Sim
( ) Não

10. Os atendimentos fisioterapêuticos dentro do NASF se restringem ao âmbito domiciliar com usuários restritos ao leito ou que requerem cuidados em reabilitação física?
( ) Sim
( ) Não

11. Ações de prevenção através de palestras e orientações também são habilidades e competências fisioterapeuta no NASF?
( ) Sim
( ) Não

12. O fisioterapeuta no NASF realiza ações de práticas integrativas e complementares, como acupuntura e práticas corporais?
( ) Sim
( ) Não

13. A formação acadêmica em fisioterapia estar voltada a reabilitação física?
( ) Sim
( ) Não

14. A modalidade de ensino EAD na fisioterapia, atribui habilidades e competências para atuação na Atenção Básica?
( ) Sim
( ) Não

15. Você se sente preparado para atuar no NASF?
( ) Sim
( ) Não 